\title{
Influence of Separated Vortex on Aerodynamic Noise of an Airfoil Blade
}

\author{
Soichi Sasaki $^{1}$, Hajime Takamatsu ${ }^{2}$, Masao Tsujino ${ }^{3}$, Haruhiro Tsubota ${ }^{3}$ \\ and Hidechito Hayashi ${ }^{1}$ \\ 1. Faculty of Engineering, Nagasaki University, 1-14 Bunkyo-machi, Nagasaki 852-8521, Japan \\ 2. Graduate School of Science and Technology, Nagasaki University, 1-14 Bunkyo-machi, Nagasaki 852-8521, Japan \\ 3. Research Division, Komatsu Ltd., 1200, Manda, Hiratsuka-shi, Kanagawa 254-8567, Japan
}

\begin{abstract}
In order to clarify the mechanism by which aerodynamic noise is generated from separated flow around an airfoil blade, the relation between the attack angle and the aerodynamic noise of the blade was analyzed using a wind tunnel experiment and a CFD code. In the case of rear surface separation, the separated vortex which has a large-scale structure in the direction of the blade chord is transformed into a structure that concentrates at the trailing edge with an increase in the attack angle. The aerodynamic noise level then becomes small according to the vortex scale in the blade chord. When the flow is separated at the leading edge, a separated vortex of low pressure is formed at the vicinity of the trailing edge. The pressure fluctuations on the blade surface at the vicinity of the trailing edge become large due to the vortex in the wake. It is considered that the aerodynamic noise level increases when the flow is separated at the leading edge because the separated vortex is causing the fluctuations due to wake vortex shedding.
\end{abstract}

\section{Keywords: Vortex, Aerodynamic Noise, Shear Flow, Separation, Airfoil}

\section{Introduction}

Since the fluid around an airfoil blade flows along the body, the blade has an advantage in that the pressure drop and slipping can be controlled in turbomachinery. It has been confirmed experimentally that the flow controlled by the airfoil blade is effective for reducing the noise of the jet fan when a symmetrical airfoil blade is employed for the impeller [1]. In a fundamental investigation of the discrete frequency noise (DFN) generated by a NACA series blade, Hayashi et al. visualized the flow around the blade using a water tunnel [2]. According to their study, it has been clarified that the DFN generated by the blade relates to the characteristics of the flow in the dead air region. On the other hand, Suzuki et al. discussed fea- tures of the aerodynamic noise in NACA0012 airfoil in the flow of several stalled conditions [3-4]. In their experiment, the existence of a separation bubble formed on the suction pressure side of the blade was demonstrated by directly measuring surface pressure fluctuations. Moreover, they have clarified that the aerodynamic noise in stalled conditions is dependent on the static pressure fluctuation of the re-attachment point on the blade surface. However, even when the fan is driven under actual operating conditions, the flow has an deviation angle from that of the blade. In such a case, the flow is totally separated from the blade surface. Therefore, the relation between such an aerodynamic noise and the separated flow becomes a major issue on the application to the fan noise. 


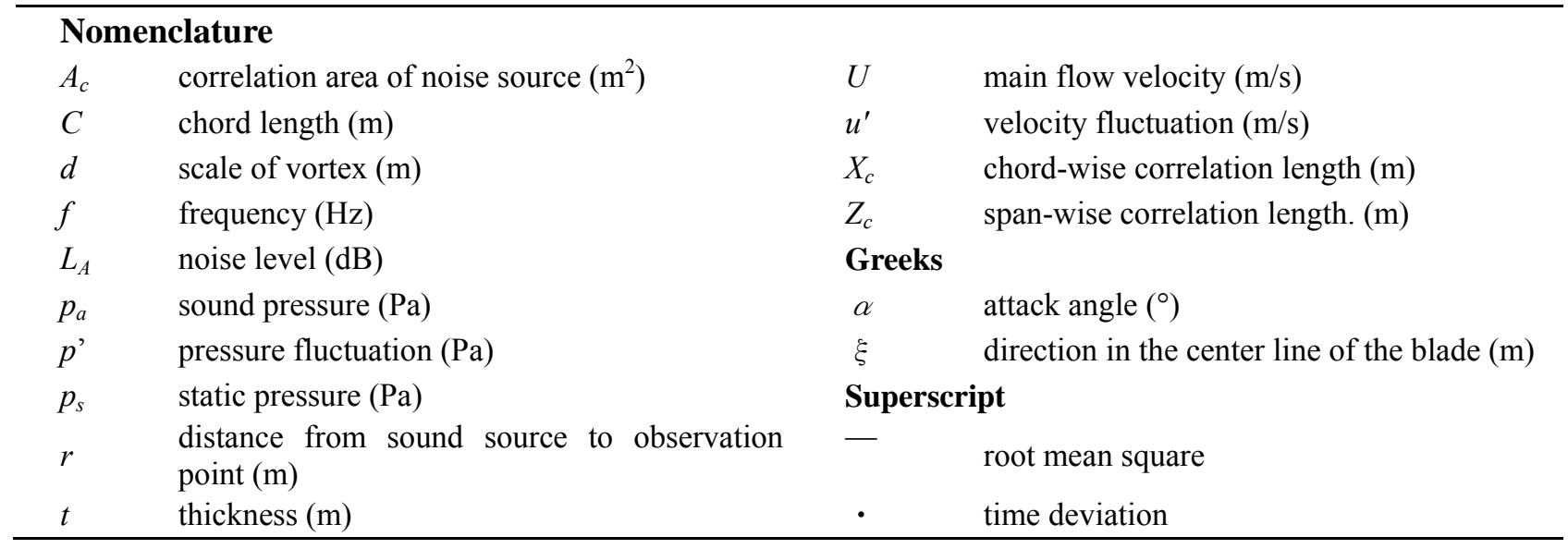

In this study, in order to clarify the mechanism by which aerodynamic noise is generated by the separated flow around the airfoil blade, the influence of the attack angle on the aerodynamic noise of the NACA00 series blade is analyzed using a wind tunnel experiment and numerical simulations.

\section{Experimental Setup}

\section{Experimental apparatus}

The airfoil blade NACA0008 used in this experiment is shown in Fig. 1. The main dimensions of the blade are listed in Table 1. Blades NACA0008 and NACA0018 were used in this experiment. The attack angle is defined as the angle formed by the center line of the airfoil and the stream line of the main flow. The two surfaces of the blade are called the "Front Surface" and "Rear Surface". Generally, the separated shear layer forms on the rear surface side of the blade.

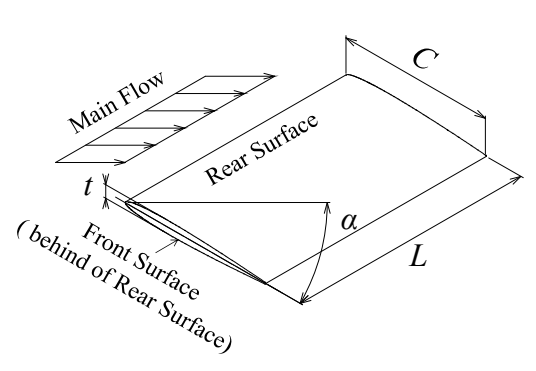

(a) schematic view

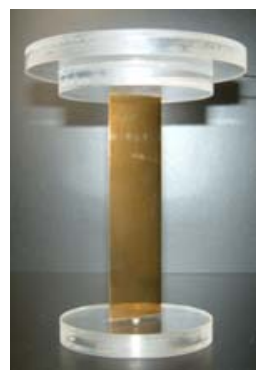

(b) test blade
Fig. 1 Configuration of the NACA0008

Table 1 Main dimensions of the NACA00 series blades

\begin{tabular}{ccc}
\hline & NACA0008 & NACA0018 \\
\hline Chord length, $C(\mathrm{~mm})$ & 30 & 30 \\
Thickness, $t(\mathrm{~mm})$ & 2.4 & 5.4 \\
$t / C$ & 0.08 & 0.18 \\
Span length, $L(\mathrm{~mm})$ & 100 & 100 \\
\hline
\end{tabular}

Figure 2 shows the measurement methods for flow and noise in the wind tunnel experiment. A uniform flow of air is jetted from a $100 \mathrm{~mm}$ square nozzle. When the Reynolds number defined by the length of the nozzle is approximately $2.0 \times 10^{5}$, the turbulence of the main flow within the measurement region is less than $0.5 \%$. The leading edge of the blade is set at $100 \mathrm{~mm}$ downstream from the nozzle exit. The hot-wire probe can be moved in three directions by a traverse machine provided with the programmed coordinate data. The velocity and velocity fluctuation of the wake measured by the I-type hot wire are converted into a voltage signal using the hot-wire anemometer; the signal is sent as input to the computer through an A/D converter. A 1/2-inch microphone is set at a distance of $0.5 \mathrm{~m}$ from the trailing edge; the angle between the main flow and the microphone is $90^{\circ}$. The signal from the noise level meter is sent as input to the

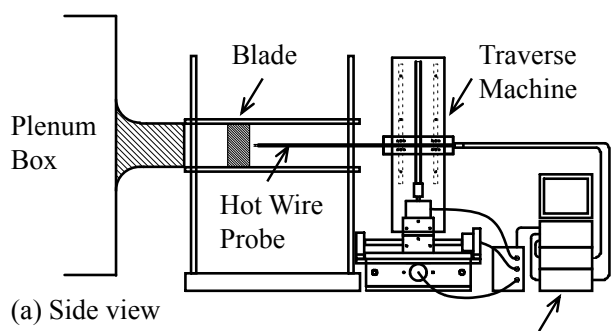

(a) Side view

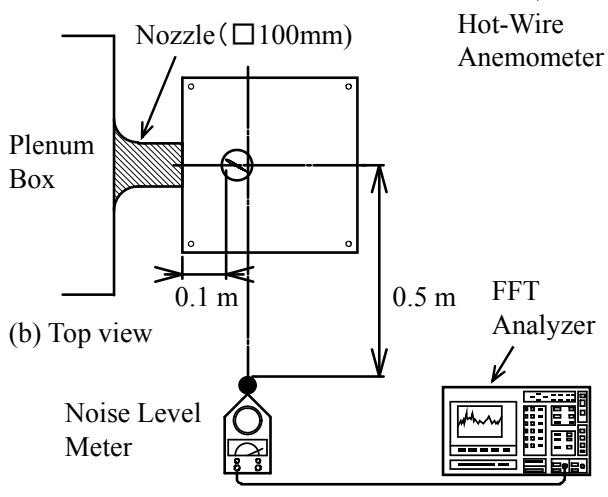

Fig. 2 Measurement device in the wind tunnel experiment 
FFT analyzer, and the frequency response characteristics of the noise is analyzed in each 1/3 octave band frequency.

\section{Numerical simulations}

The unstructured grid around NACA0008 in a numerical simulation is shown in Fig. 3.The normalized distance $y^{+}<10$ is given to the minimum grid width to express the turbulent boundary layer on the wall surface. The number of computational grid elements to solve the flow of one blade is approximately 500,000. SCRYU/ Tetra (Software Cradle Co., Ltd.) is used for the CFD code. The SIMPLEC method is used for pressure correction, and the SST model is employed for the turbulence model. The number of iterations for the unsteady flow simulation is 10,000 . The influence of the departure vortex is removed in the analysis of the periodic fluctuation phenomenon.

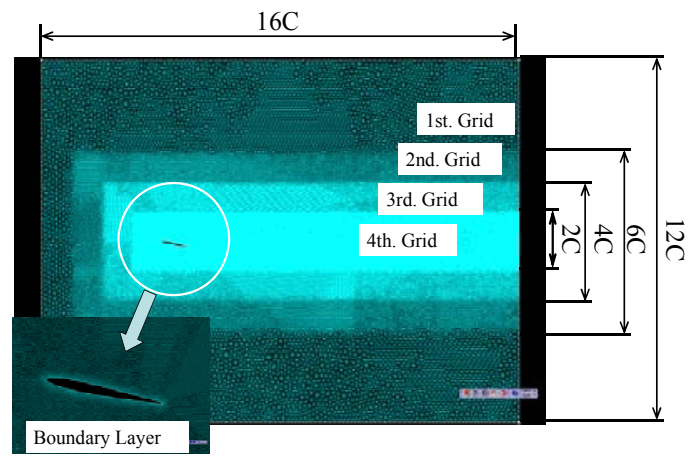

Fig. 3 Unstructured grid around NACA0008

For instance, in reference [5], the aerodynamic noise generated from a flat plate blade has been given as:

$$
\overline{p_{a}{ }^{2}} \cong\left(\frac{\cos \varphi}{4 \pi r a}\right)^{2} \int_{A} A_{c}(x, z) \overline{\dot{p}_{s}(x, z)^{2}} d A
$$

Then

$$
A_{c}(x, z)=(\pi / 4) X_{c}(x, z) Z_{c}(x, z)
$$

where, $A_{c}$ is the correlation area of the noise source, $p_{s}$ is the pressure fluctuation on the blade surface, $X_{c}$ is the chord-wise correlation length, and $Z_{c}$ is the span-wise correlation length. According to this expression, the aerodynamic noise relates to the pressure fluctuation and the correlation length of the noise source. Generally, the chord-wise correlation length is determined by the two-dimensional scale of the vortex; the span-wise correlation length is estimated by its three-dimensional scale.

\section{Results and Discussion}

Figure 4 shows the relation between the attack angle and the overall noise level. The noise level of NACA 0008 decreases from $12^{\circ}$ to $20^{\circ}$; it increases from $28^{\circ}$ to $36^{\circ}$. In comparing the two blades, the influence of the blade thickness ratio on the noise characteristics of the separated flow at large attack angles is small. Moreover, it is clear that the aerodynamic noise generated from the blade does not simply increase monotonically with the attack angle. Based on the flow pattern around the blade obtained by the wind tunnel experiment, in the explanation that follows, the flows at attack angles ranging from $12^{\circ}$ to $20^{\circ}$ will be called the "Rear Surface Separation (RSS)" while those at attack angles ranging from $28^{\circ}$ to $36^{\circ}$ will be referred to as the "Leading Edge Separation (LES)."

The spectral distributions of aerodynamic noise generated from the two types of blade are compared in Fig. 5. This figure shows the spectra at the attack angle where the noise level becomes maximal in Fig. 4. The background noise of the wind tunnel is shown as the broken line. Overall noise level is almost identical for the two blades $(86.1 \mathrm{~dB})$. The frequency at which the noise is maximal is higher for NACA0008 than for NACA0018. It is possible that a Karman vortex street with high frequency is shed into the wake because the width of the wake in NACA0008 is narrower than that in NACA0018. The characteristics of the discrete frequency noise in the vicinity of this attack angle have already been studied in

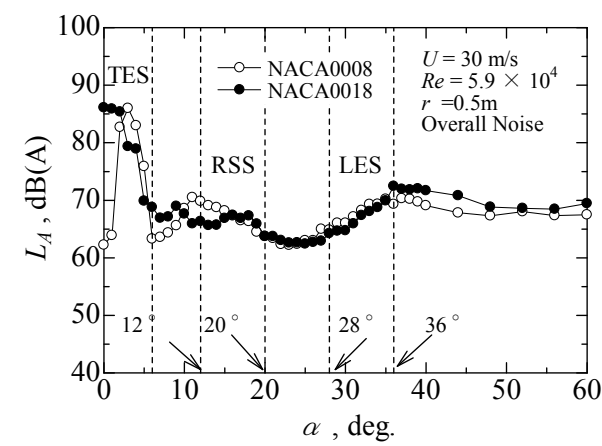

Fig. 4 Relation between the attack angle and the overall noise level

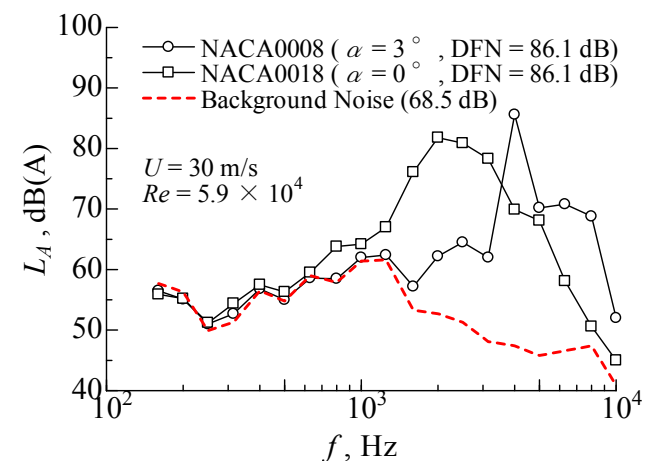

Fig. 5 Comparison of the spectral distributions for discrete frequency noise 
reference [2]. Thus, the characteristics of the discrete frequency noise are not discussed in this paper.

Comparisons of the spectral distribution of noise at different attack angles are presented in Fig. 6. In the case of rear surface separation, the broadband noise level distributed to $2000 \mathrm{~Hz}$ or more at an attack angle of $20^{\circ}$ becomes smaller than that at an angle of $12^{\circ}$. On the other hand, the broadband noise becomes larger when the flow pattern changes to leading edge separation. At attack angles of $12^{\circ}, 28^{\circ}$, and $36^{\circ}$, a discrete frequency noise due to wake vortex shedding can be recognized. In this spectral distribution, it can be confirmed that not only the broadband noise, but also the discrete frequency noise, becomes similar to the characteristic overall noise level.

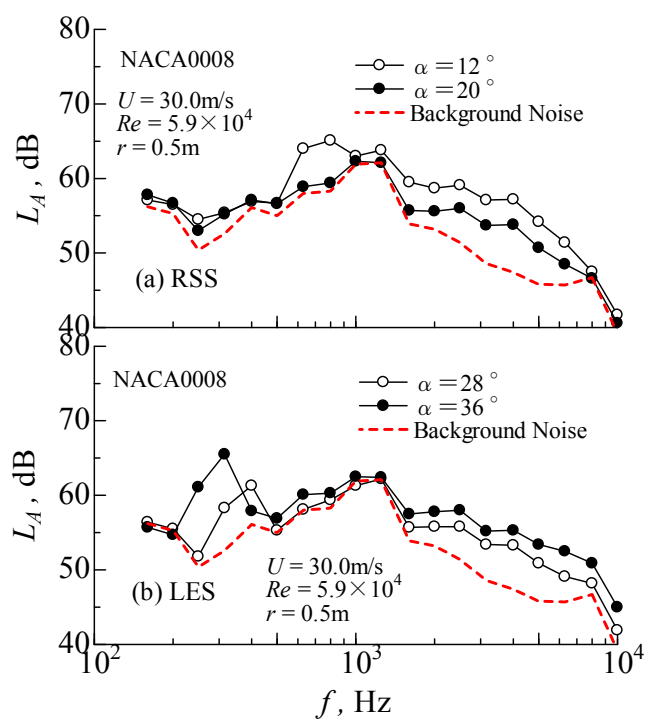

Fig. 6 Comparison of the spectral distribution of noise at different attack angles

Figure 7 shows the measured flow pattern on the rear surface of NACA0008. The attack angle is $10^{\circ}$. In Fig. 7(a), an area with high velocity is formed in the vicinity of the leading edge. The velocity fluctuation, shown in Fig. 7(b), becomes large in the vicinity of the high velocity domain on the rear surface. It can be recognized that the aerodynamic noise source at this attack angle exists on the rear surface. The separated flow in the case of an attack angle of $20^{\circ}$ is shown in Fig. 8. The flow separates from the leading edge, creating a separated shear layer on the rear surface. The velocity fluctuation becomes strong in the separated shear layer from the leading edge to trailing edge. At this attack angle, the velocity fluctuation in the shear layer is larger than the fluctuation on the blade surface.

The distribution of calculated mean velocities around the blade is shown in Fig. 9. The velocity distribution can represent the high velocity domain at the leading edge and the separated shear layer on the rear surface of the blade (see Fig. 7(a)). The measured and calculated flows in the wake are compared in Fig. 10. The attack angle is set at $10^{\circ}$. The circular symbols represent measured values and the solid line shows the calculated values. The velocity distribution of the numerical simulation can represent the width of the wake and the diffusion of the actual measured flow.

The mean pressure around the blade obtained by CFD is shown in Fig. 11. At an attack angle of $12^{\circ}$, a domain of low pressure generated by the separated vortex is formed on the rear surface. At an attack angle of $20^{\circ}$, a pressure domain is formed at the vicinity of the trailing

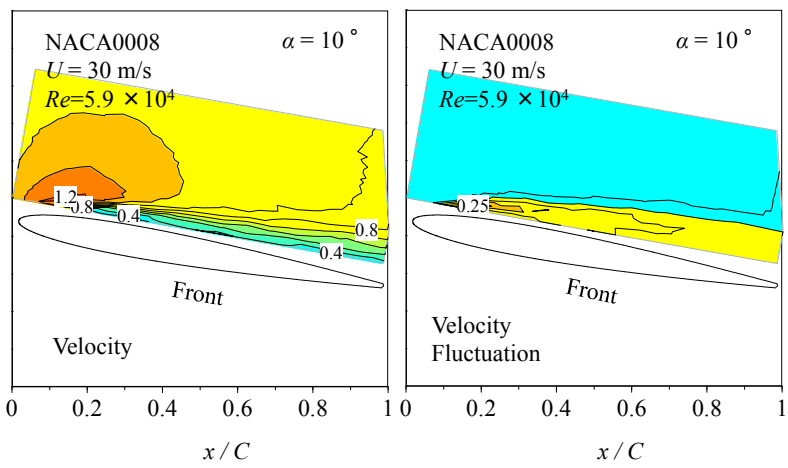

(a) Velocity

(b) Velocity fluctuation

Fig. 7 Measured flow pattern on rear surface at $\alpha=10^{\circ}$

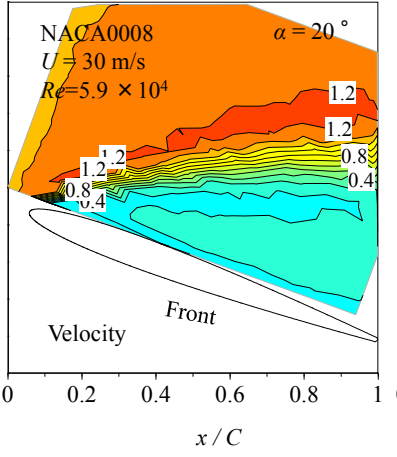

(a) Velocity

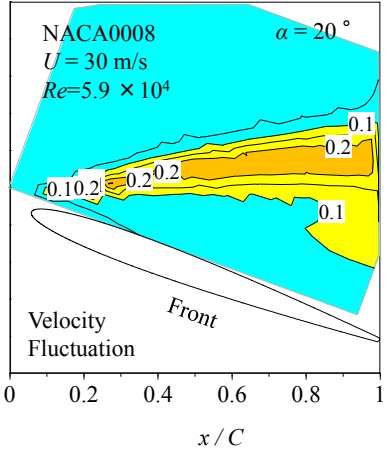

(b) Velocity fluctuation
Fig. 8 Measured flow pattern on rear surface at $\alpha=20^{\circ}$

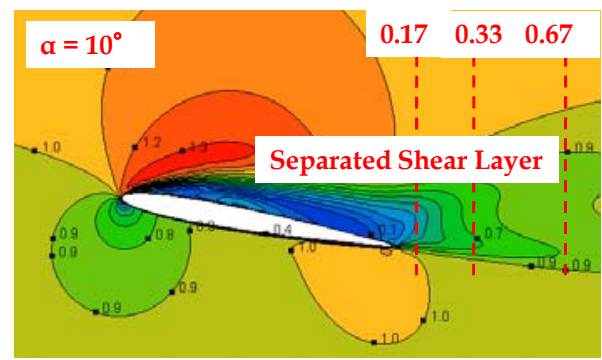

Fig. 9 Distribution of calculated mean velocities around the blade 


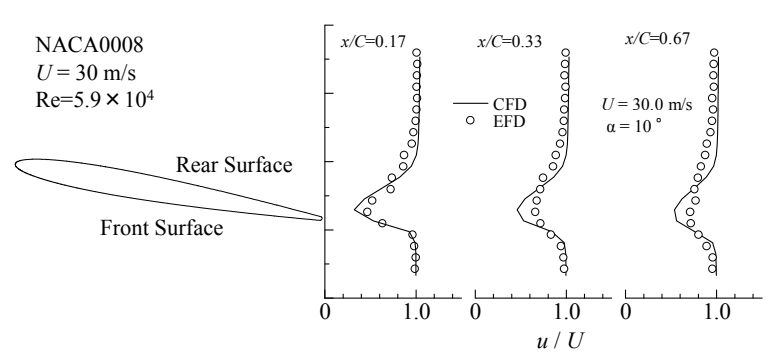

Fig. 10 Comparison of the measured and calculated flows in the wake

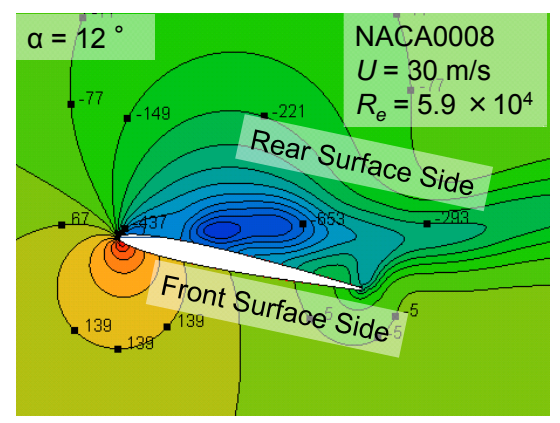

(a) $\alpha=12^{\circ}$

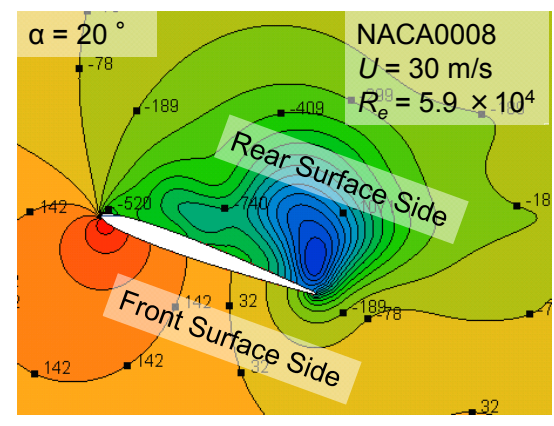

(b) $\alpha=20^{\circ}$

Fig. 11 Calculated mean pressure around the blade in the case of rear surface separation

edge. It can be noticed that the flow pattern related to the aerodynamic noise is shifted from the rear surface to the trailing edge with an increase in the attack angle that causes rear surface separation.

The distribution of pressure fluctuation on the blade surface is compared in Fig. 12. The horizontal axis shows the position along the center line of the blade from the leading edge normalized by the chord length. The pressure fluctuation on the rear surface is larger than that of the fluctuation of the front surface. The domain of large pressure fluctuation at an attack angle of $12^{\circ}$ is more extensive than that at an angle of $20^{\circ}$. According to Fig. 11, it is possible to consider that these pressure fluctuations are generated by the separated vortex on the rear surface. A large-scale separated vortex aligned along the direction of the blade chord is transformed into a structure that is concentrated at the trailing edge when the attack angle increases in the region of rear surface separation. It is considered that the aerodynamic noise in the state of the rear surface separation is reduced by the scale of the vortex in the direction of the blade chord.

The measured velocity fluctuation around the blade in the state of leading edge separation is compared in Fig.13. In Fig. 13(a) and Fig 13(b), the attack angles are $20^{\circ}$ and $40^{\circ}$, respectively. Since a symmetrical swirl of velocity fluctuation is formed in the wake, it is considered that a Karman vortex street is shed into the wake in each attack angle. The velocity fluctuation becomes larger at positions in which the vortex swirls in the wake. Therefore,

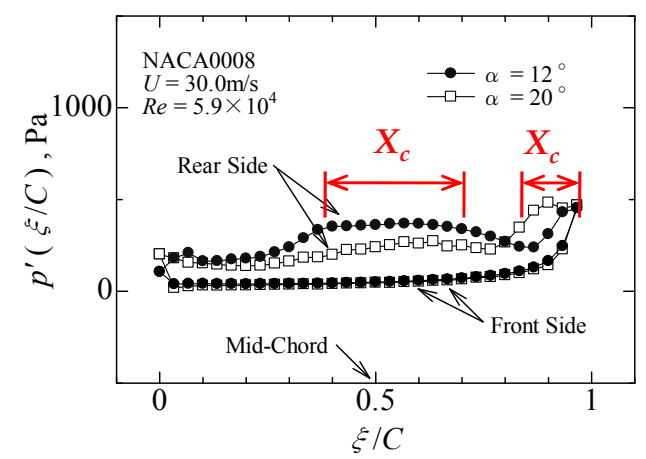

Fig. 12 Comparison of pressure fluctuation on the blade surface in the case of rear surface separation

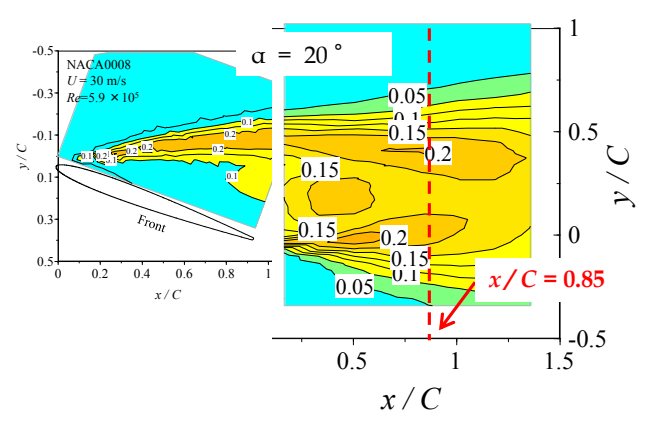

(a) $\alpha=20^{\circ}$

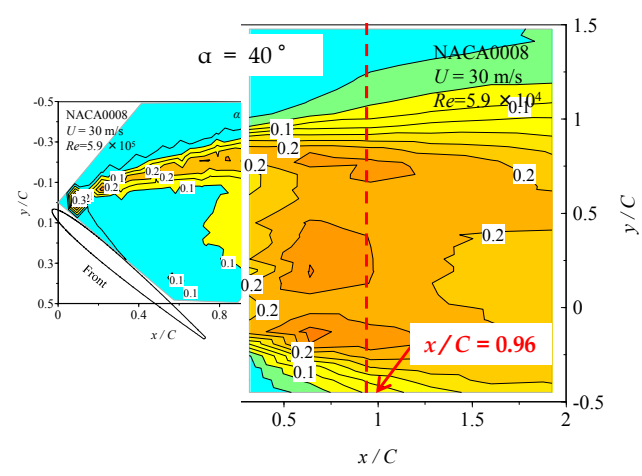

(b) $\alpha=40^{\circ}$

Fig. 13 Comparison of the distribution of measured velocity fluctuation 
the wake vortex becomes one of the factors comprising an aerodynamic noise source. The distribution of velocity fluctuation in the vertical direction in relation to the main flow is shown in Fig. 14. The position of the measured section is the point at which the velocity fluctuation becomes maximal in the wake (broken line in Fig. 13). The wake characteristics based on the distributions of velocity fluctuation are summarized in Table 2 . The scale of the vortex is estimated by the half width of the distribution. The maximum value of the velocity fluctuation at an attack angle of $40^{\circ}$ increases from that at an angle of $20^{\circ}$ by $14.8 \%$. On the other hand, the scale of the vortex at an attack angle of $40^{\circ}$ becomes $51.0 \%$ larger than that an angle of $20^{\circ}$. Even if the kinetic energy concerning the sound pressure of the aerodynamic noise is estimated by the square of the maximum velocity fluctuation (i.e. double the ratio of the increase in fluctuation), the influence of the scale of the vortex becomes a major factor in the aerodynamic noise source under conditions of leading edge separation.

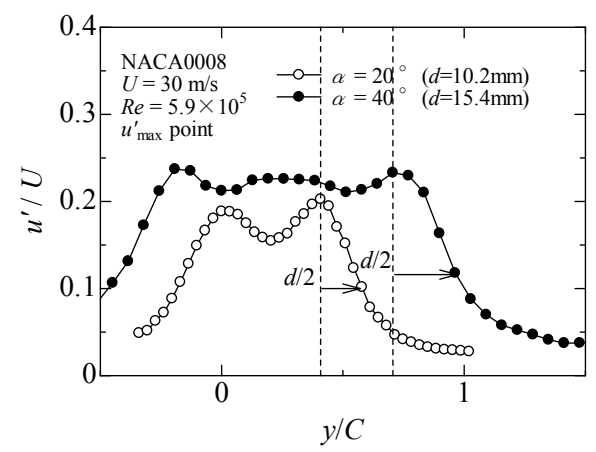

Fig. 14 Distribution of the velocity fluctuation in the wake

Table 2 Wake characteristics of NACA blades

\begin{tabular}{ccc}
\hline & NACA0008 & NACA0018 \\
\hline Velocity fluctuation, $u^{\prime} / U$ & 0.203 & 0.233 \\
Scale of vortex, $d(\mathrm{~mm})$ & 10.2 & 15.4 \\
\hline
\end{tabular}

The mean pressure distributions around the blade at attack angles of $28^{\circ}$ and $36^{\circ}$ are shown in Fig. 15. At each attack angle, a separated vortex of low pressure is formed at the vicinity of the trailing edge of the blade. It is considered that the aerodynamic noise caused by leading edge separation is influenced by the separated vortex formed at the trailing edge.

The distribution of pressure fluctuation around the blade is shown in Fig. 16. A domain of the pressure fluctuation distribution at an attack angle of $36^{\circ}$ is more extensive than that at an angle of $28^{\circ}$. In the case of leading edge separation, it is clear that one of the reasons for the increased noise level is the scale of the vortex at the trailing edge. It is considered that the noise source in- duced by the separated vortex at the vicinity of trailing edge is formed by the fluctuation of the vortex shedding in the wake.

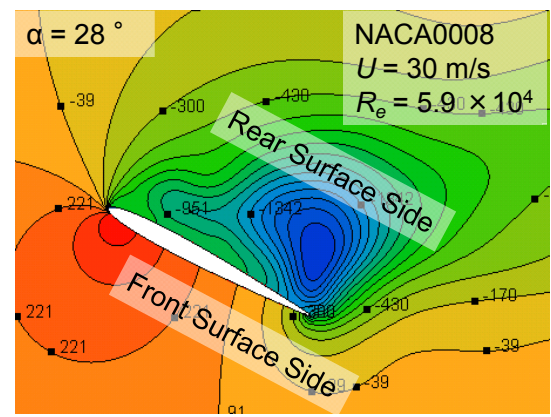

(a) $\alpha=28^{\circ}$

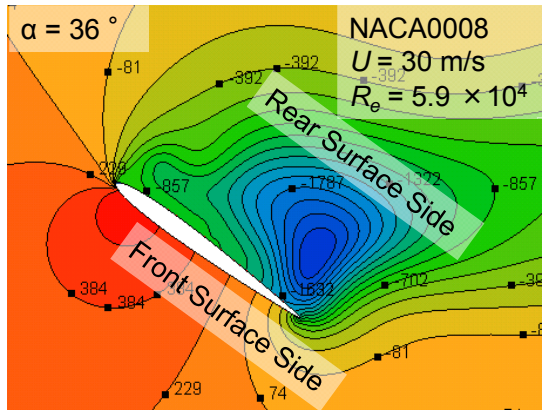

(b) $\alpha=36^{\circ}$

Fig. 15 Calculated mean pressure around the blade in the case of leading edge separation

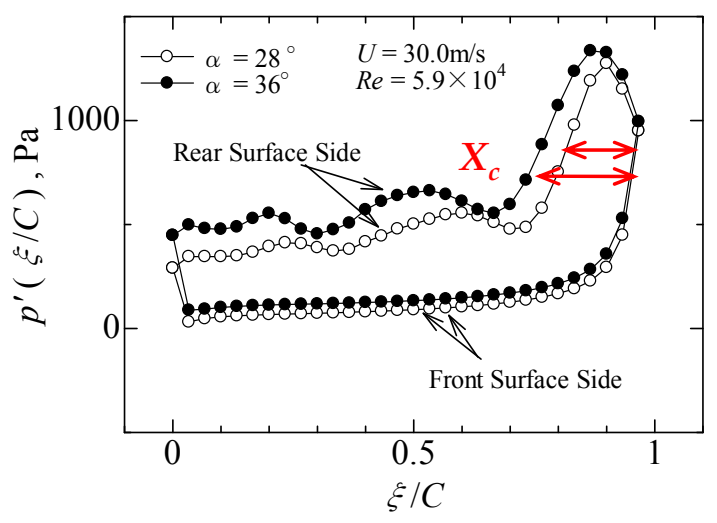

Fig. 16 Comparison of pressure fluctuation distributions on the blade surface in the case of leading edge separation

\section{Conclusion}

From the results of analyses on the relation between the attack angle and the aerodynamic noise of the airfoil blade, the following conclusions are derived.

The aerodynamic noise generated from the blade became small when the flow separated from the rear surface 
of the blade with an increase in the attack angle; the noise increased with an increase in the attack angle when the flow separated from the leading edge. The influence of the blade thickness ratio on the noise characteristics was small.

In the case of rear surface separation, the velocity fluctuation at the vicinity of the separated vortex formed on the rear surface of the blade became large. The large-scale separated vortex aligned along the direction of the blade chord was transformed into a structure concentrated at the trailing edge with an increase in the attack angle. In this case the aerodynamic noise level became small in accord with the scale of the vortex along the blade chord.

A separated vortex of low pressure was formed at the vicinity of the trailing edge when the flow was separated at the leading edge. The pressure fluctuation on the blade surface at the vicinity of trailing edge became large due to the vortex in the wake. When the flow was separated at the leading edge, the aerodynamic noise level was increased by a separated vortex that caused fluctuations due to wake vortex shedding.

\section{References}

[1] Kodama, Y., Hayashi, H., Murayama, M., Kondou, S. and Yamaguchi, H, (2005), Reduction of Jet Fan Noise, Turbomachinery (in Japanese), 33 (2), 100-108.

[2] Hayashi, H., Kodama, Y., and Sasaki, S., (1999), Relationship between the Discrete Frequency Noise and Wake Vortices on Symmetrical Airfoil, Journal Visualization Society of Japan (in Japanese), 19 (72), 42-47.

[3] Suzuki, Y., Kato, C., Suzuki, T., and Fujita, H., (2007), Aerodynamic and Aeroacoustical Characteristics of Low Reynolds Number Two- Dimensional Airfoil Flows, Transactions of the Japan Society of Mechanical Engineers (B) (in Japanese), 73(736), 2476-2486.

[4] Suzuki, Y., Kato, C., Miyazawa, M., and Fujita, H., (2007), Analysis of Aerodynamic Sound Sources in a Flow around a Two-Dimensional Airfoil under Stalled Conditions, Transactions of the Japan Society of Mechanical Engineers (B) (in Japanese), 73(736), 2487-2497.

[5] Maruta, Y. and Kotake, S., (1983), Separated Flow Noise of a Flat Plate at Large Attack Angle, Journal of Sound and Vibration, 89(3), 335-357. 